\title{
Corrections and clarifications
}

Minerva

A recent updating of our electronic processing system seems to have failed to allow for the possibility that contributors of a Minerva picture might work at the same address but in different departments. Thus, several authors' names did not appear with the Minerva photograph in the issue of 26 October (p 978): Stephen H Boyce (staff grade), Alasdair R Corfield (specialist registrar), Crawford McGuffie (consultant), and James Stevenson (consultant) are all located at the accident and emergency department, Crosshouse Hospital, Kilmarnock KA2 0BE.

Removal of radiation dose response effects: an example of over-matching

The authors of this Education and debate article, J L Marsh, J L Hutton, and Keith Binks, have confirmed that the equation in the section headed "Method: analysis of a matched case-control study" contained an error (10 August, p 327-30). "Exp" should have appeared before the $\beta$ symbol in the conditional likelihood.

Editors pledge support for African journals

In the last paragraph of this article by Tessa Richards in the "roundup" section of the News (26 October, p 922-3), "Latin-American medical journals online" should have been given its correct title of "SciELO-Scientific Electronic Library Online." 